Yoram Koren
Professor.
Fellow ASME

Tsu-Ren Ko

Research Assistant.

\section{A. Galip Ulsoy}

Associate Professor.

Mem. ASME

Department of Mechanical Engineering and Applied Mechanics

The University of Michigan,

Ann Arbor, MI 48109-2125

Kourosh Danai

Assistant Professor,

Department of Mechanical Engineering,

The University of Massachusetts,

Amherst, MA

\title{
Flank Wear Estimation Under Varying Cutting Conditions
}

A model-based methodology, designed to operate under varying cutting conditions, for on-line estimation of flank-wear rate based on cutting force measurements is introduced. The key idea is to employ a model of the relationship between force and flank wear, together with on-line parameter estimation methods. This permits separation of the direct effect of changing cutting conditions on force from the indirect effect where changing cutting conditions affect the wear which, in turn, affects the force. Simulation results confirm the effectiveness of this strategy for turning with varying speed, feed, or depth of cut. Experiments, conducted for turning operations with a varying depth of cut, show good agreement between estimated wear values and the actual values of tool wear measured intermittently during the cut.

\section{Introduction}

On-line tool wear estimation in machining has been investigated by numerous researchers (e.g., [1-3]). Yet, despite years of research in this area, a reliable on-line tool wear measurement technique does not exist $[4,5]$. Direct measurement of tool wear using optical methods can only be employed when the tool is not in contact with the workpiece [6]. Radiometric techniques as direct measurements have not proven to be practical for production environments [7-8]. Indirect methods which rely on the relationship between tool wear and a measurable signal (e.g., force, torque, acoustic emission, temperature, and vibrations) to estimate the wear have also been extensively studied [9-17].

Most of the indirect approaches have been developed for fixed cutting conditions. In practical applications, however, the cutting conditions are not fixed. The depth of cut changes because of the part geometry, as shown for example in Fig. 1 , and the feed might change according to control strategies (e.g., reducing the feed before approaching corner points to avoid overcut). Therefore, a wear estimation strategy that operates under varying cutting conditions is needed.

This paper uses cutting force measurements to estimate the flank wear. The force depends on the cutting variables: the cutting speed, $v$, the feed, $f$, and the depth of cut, $d$, as well as on the tool wear $W$, namely $F(v, f, d, W)$. The wear itself also depends on the cutting variables, i.e., $W(v, f, d)$. Since the measured force is affected by both tool wear and the changing cutting conditions, and the wear itself depends on the

Contributed by the Dynamic Systems and Control Division for publication in the Journal of Dynamic Systems, Measurement, and Control. Manuscript received by the Dynamic Systems and Control Division November 1989; revised manuscript received September, 1990. Associate Editor: H. Kazerooni

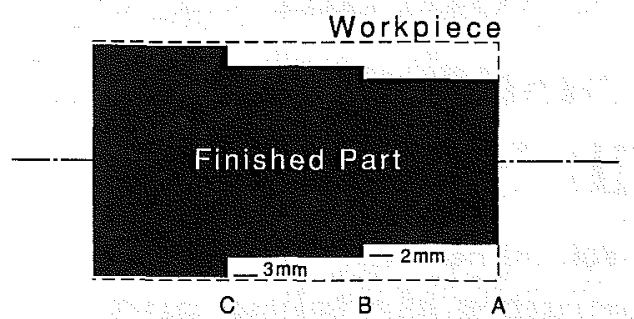

Fig. 1 In a typical part program the first chip is cut with $d=1 \mathrm{~mm}$ along the part, then $d=3 \mathrm{~mm}$ from $A$ to $C$; finally $d=2 \mathrm{~mm}$ from $A$ to $B$. A complex part includes many such variations in the depth of cut.

cutting conditions, any change in a cutting condition affects the force measurement both directly and also indirectly through the wear. The problem is to separate the direct effect of the wear on the force measurement from the effect due to changing cutting variables. So far, this problem has not been thoroughly addressed in the literature.

In this paper a method is presented for the on-line estimation of flank wear in turning operations from force measurements under conditions where one of the cutting variables (i.e., depth or feed or speed) exhibits stepwise variations. The key idea in the method is to employ an on-line parameter estimation method together with a simple model of the relationship between the measured force and the flank wear. Simulation studies are presented to illustrate the use and effectiveness of the method for three cases: (i) speed $(v)$ varies, (ii) depth-of-cut (d) varies, and (iii) feed $(f)$ varies. The most common case is (ii), and experimental studies are also presented for that case. The results of the experiments show that the method can be effectively employed in practice. 


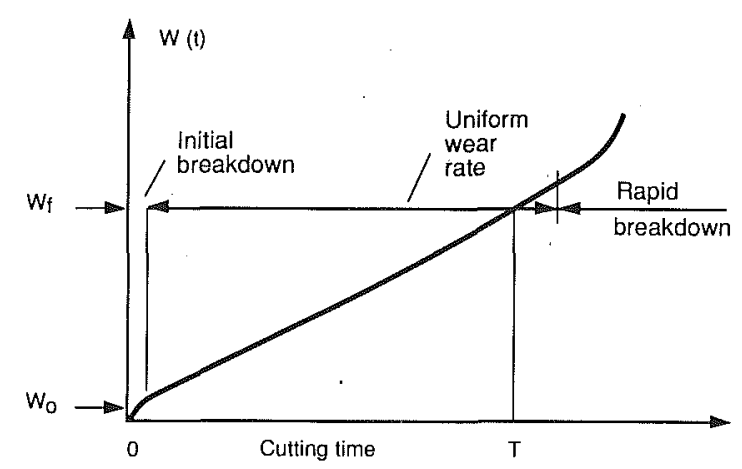

Fig. 2 Development of flank wear with time for a carbide tool

\section{The Model}

The effect of the cutting variables $d, f$, and $v$ on each of the three force components, for a sharp tool, can be expressed in terms of the following empirical relationship [18]:

$$
F_{0}^{*}=K_{s} d^{\alpha_{3}} f^{\alpha_{2}} v^{\alpha_{1}}
$$

where $K_{s}, \alpha_{1}, \alpha_{2}$, and $\alpha_{3}$ depend on the tool geometry and workpiece material. Typical values for the tangential (main) force component when cutting steel are $\alpha_{3} \approx 1, \alpha_{2} \approx 0.7$ and $\alpha_{1} \approx-0.1$ [11]. The physical analysis of the cutting process shows that the tangential force is proportional to the chip load which is, in turn, proportional to $d$ and $f$ [18]. The dependence on $v$ is not strong (i.e., $\alpha_{1}$ is small) and might be explained by changes of the work material properties due to cutting temperature variations with cutting speed. Other force components (i.e., feed and radial components) have different $\alpha_{i}$ values in equation (1). The following analysis is appropriate for any of the three force components; and for simplicity only the term force is used henceforth. The tangential (main) force component is used in all the experiments and simulations presented below.

The growth of the wear on the flank face of the tool consists of three distinct stages, as shown in Fig. 2: a short initial region of rapid wear, an approximately constant wear-rate region, and finally a very rapid wear-rate region which indicates tool failure. In practice, the tool is replaced during the second stage. Therefore, during most of the cutting process the flank wear may be approximated by

$$
W=W_{0}+\dot{W} t
$$

where $\dot{W}$ represents the wear-rate in the second region, $W_{0}$ is the intercept with the wear axis, and $t$ is time. This linear relationship results from assuming a model in which the normal force $N$ on the tool's flank face is proportional to the flank wear in the second region and is supported by experimental results [19-21]. When flank wear starts to develop, the rubbing mechanism causes a force increase, $\Delta F^{*}$. Since this rubbing force $\Delta F^{*}$ is proportional to $N$ (related by a friction coefficient), and $N$ is proportional to $W$, the total force becomes

$$
F=F_{0}^{*}+K W
$$

This linear relationship has been verified by experiments [ 21 , 22]. Theoretically, $K$ should be proportional to the depth of cut $d$, since the rubbing area is $W d$. However, for generality we assume that the feed $f$, and even the cutting speed $v$, may also affect $\Delta F^{*}$. Therefore, we assume that

$$
K=K(v, f, d)=C_{p} v^{\beta_{1}} f^{\beta_{2}} d^{\beta_{3}}
$$

Typically, $\beta_{3}>\beta_{2}>\beta_{1}$. Our experiments with carbide tools show that $\beta_{3} \simeq 1,0.4>\beta_{2}>0$, and $\beta_{1} \approx 0$ [22].

The flank wear can be represented by utilizing the extended Taylor tool life equation

$$
T v^{\gamma_{1}} f^{\gamma_{2}} d^{\gamma_{3}} C_{n}=1
$$

where $C_{n}, \gamma_{1}, \gamma_{2}$, and $\gamma_{3}$ depend on the workpiece material and the tool's material and geometry. The tool life $T$ may be defined by a final flank wear $W(T)=W_{f}$ at which the tool is replaced. Combining equations (2) and (5) yields

$$
\dot{W}=\left(W_{f}-W_{0}\right) C_{n} v^{\gamma_{1}} f^{\gamma_{2}} d^{\gamma_{3}}
$$

where typically, $\gamma_{1}>\gamma_{2}>\gamma_{3}$. For example, in cutting steel with uncoated carbide tools, $\gamma_{1} \approx 3, \gamma_{2} \approx 1$, and $\gamma_{3} \approx 0.3$ $[18,21]$.

Combining equations (2) and (3) yields

$$
F=F_{0}^{*}+K W_{0}+K \dot{W} t
$$

The initial wear develops rapidly in the first few seconds of the cut [23], and experience shows that it is not possible to measure the initial force level $F_{0}^{*}$ or the sharp tool. In practice, we measure an initial force $F_{0}=F_{0}^{*}+K W_{0}$. Consequently, the additional measured force component due to wear is $\Delta F$ $=K \dot{W} t$, or explicitly from equations (4) and (6)

$$
\Delta F=C_{p} v^{\beta_{1}} f^{\beta_{2}} d^{\beta_{3}} \dot{W}\left(v^{\gamma_{1}}, f^{\gamma_{2}}, d^{\gamma_{3}}\right) t
$$

The wear rate is expressed in equation (8) as a function of the cutting variables. If the validity of the Taylor tool life equations had been assumed, then equation (6) could have been substituted into equation (8). Instead, we consider the use of estimation methods to obtain the wear rate from force measurements.

We will consider the case where the wear rate depends on the cutting conditions, but only one of the cutting variables varies. Then equation (8) can be written as

$$
\Delta F=C b^{\beta} \dot{W}\left(b^{\gamma}\right) t
$$

where $b$ is the particular cutting variable which varies (i.e., $b$ might be $v, f$, or $d$ ). The coefficient $C$ depends on the tool and workpiece material as well as on the other cutting variables that do not vary. A change in $b$ has both a direct effect on the force measurement through equation (1) and $b^{\beta}$ in equation (9), and an indirect effect through the change in the wear rate $W$. The challenge is to separate the two effects by using estimation techniques. In principle three possible cases exist for equation (9): case \#(1) $\beta \simeq 0$ and $\gamma \neq 0$, or case $\#(2) \beta \neq 0$ and $\gamma \simeq 0$, or case $\#(3) \beta \neq 0$ and $\gamma \neq 0$.

The first two cases are special cases of the third, but it will be shown below that they can use more reliable estimation techniques and thereby provide better results. Approximations could be made in the model given by equations (1), (4), and (6) to justify the utilization of the more reliable estimation methods for cases 1 and 2. In practice, the benefits of using reliable estimation techniques with approximate models must be weighed against the use of a complete model with an estimation technique that is sensitive to some measurements.

A typical example for the first case is when $b=v$; changes in the cutting speed strongly affect the wear rate, but their direct effect on $\Delta F$ is small [24]. A practical example of case $\# 2$ is when $b=d$; the effect of the depth of cut on flank wear is very small [25], but it is almost linearly proportional to $\Delta F$ $[21,26]$. The case where both exponents in equation (9) are nonzero (case \#3) is the most general and most complicated one, since the effect of the variations in $b$ are felt both directly, through $b^{\beta}$, and indirectly, through the wear rate. In practice, $b=f$ and sometimes $b=d$ correspond to this case $[18,22]$.

In this research, we consider the cases when the cutting variable $k$ changes step-wise with values $b_{k}$. A step change from $b_{k-1}$ to $b_{k}$ at time $t_{k}$ will cause an abrupt change in the cutting force from $F_{k-1}^{\prime}$ to $F_{k}$, as shown in Fig. 3. This cutting force change is sensitive to the variations in $d$ and $f$, but not to the variations in $v$.

The interval $k$ is defined here as the $k$ th segment of a cut in which the cutting variable $b_{k}$ is kept constant. (Note that the measured cutting force is sampled at a constant rate and on our experimental system the sampling rate is $2 \mathrm{~Hz}$.) The initial force at interval $k, F_{k}\left(b_{k}, t_{k}\right)$, or simply $F_{k}$, might be 


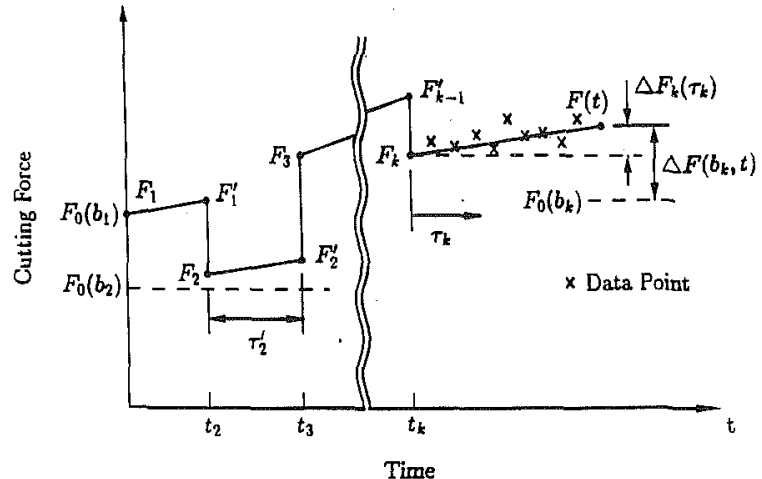

Fig. 3 Schematic of the cutting force, $F$, for piecewise constant changes in the cutting condition, $b$

considered as a reference point for the segment. The force increase during the interval $k, \Delta F_{k}\left(b_{k}, \tau_{k}\right)$, or simply $\Delta F_{k i}$, is calculated at every sampling period $i$ from

$$
\Delta F_{k i}=F_{i}\left(b_{k}\right)-F_{k}\left(b_{k}\right)
$$

where $F_{i}\left(b_{k}\right)$ is the measured force at the $i$ th period. The discrete-time version of equation (9) becomes

$$
\frac{\Delta F_{k i}}{\tau_{k}}=X\left(b_{k}^{\gamma}\right) b_{k}^{\beta}
$$

where $\tau_{k}=t-t_{k}$, and

$$
X\left(b^{\gamma}\right)=C \dot{W}\left(b^{\gamma}\right)
$$

With these notations in mind, three tool wear estimation methods corresponding to the three possible modeling cases discussed above will be introduced.

\section{Analysis}

This section provides a detailed description of the three estimation methods (referred to as Methods I, II, and III) and studies their performance using simulation.

For the first two cases, the estimation is done by first computing the variable $X$ from the rate of the cutting force increase during cutting, as given by equation (11). The quantity $C W$ is then estimated by approximately integrating $X$ at each sampling period from the equation

$$
\widehat{C W}(t)=C W_{0}+\left(\sum_{j=1}^{k-1} \widehat{C W_{j}}\right)+\hat{X}_{k} \tau_{k}
$$

where $\widehat{C W_{j}}=\hat{X}_{j} \tau_{j}^{\prime}$ with $\tau_{j}^{\prime}$ being the total time elapsed during interval $j ; \tau_{k}=k \Delta t$. where $\Delta t=0.5$ second is the sampling period, and $t=\tau_{k}+\Sigma_{j=1}^{k-1} \tau_{j}^{\prime}$. Note that $\hat{X}_{j}$ indicates the estimated rate of tool wear, and the actual tool wear can only be estimated from $\hat{C W}$ when $C$ is known.

Equation (11), however, cannot be used in the third case (i.e., where both $\beta \neq \neq 0$ and $\gamma \neq 0$ ). In this case, Method III uses the force increase from the initial engagement $\Delta F\left(b_{k}, t\right)$, rather than $\Delta F_{k}\left(b_{k}, \tau_{k}\right)$ (see Fig. 3 for the definition of $t$ and $\left.\tau_{k}\right)$. Therefore, the model parameters for $F_{0}\left(b_{k}\right)$ must be estimated in addition to the parameters of $\Delta F$.

Methods I and II do not rely on a mathematical model of $F_{0}$ for estimation, and therefore random variations in $F_{0}$ (or errors in the estimated $F_{0}$ ) will not affect the estimation of the wear rate. Method II has initial estimation errors. Therefore, it also includes a real-time reliability test that must be met before we accept the estimation results. Method III, however, is sensitive to errors in the estimation of $F_{0}$. Therefore, applying the first two approaches is preferred wherever justified (i.e., whenever we can reasonably assume that either $\beta$ or $\gamma$ is zero in equation (9)).

3.1 Method I. Method I is based upon a standard esti-

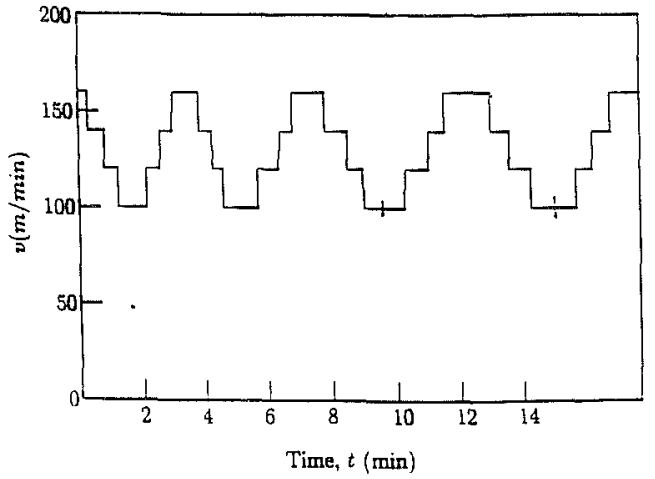

Fig. 4(a) Step changes in the cutting speed, $v$, for use in Method I

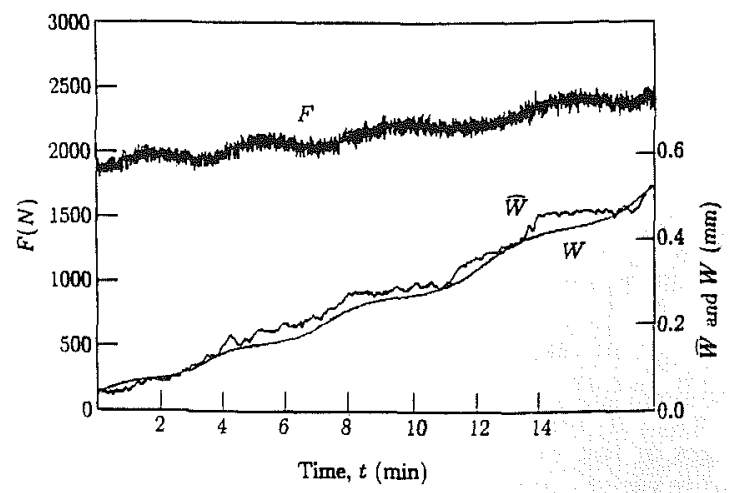

Fig. $4(b)$ The simulated cutting force, $F$, the estimated wear, $W$, and the true wear, $W$ for Method I

mation algorithm and is included here for completeness of the methodology and as an introduction to the other methods.

In this method, the corresponding cutting forces $F_{k}\left(b_{k}\right)$ at $t_{k}$ (the time when abrupt changes in $b$ take place) are removed from the total cutting force at each interval $k$, and the resultant signal $\Delta F_{k}$ is analyzed. A direct measurement of these constant forces at $t=t_{k}$ is unreliable because of noise and possible overshoot. In our experiments for the changes at $t_{k}$ the force signal was observed to oscillate, overshoot or undershoot. Errors in the measurement of $F_{k}\left(b_{k}\right)$ at the beginning of the interval cause severe errors in $\Delta F_{k}$ and, in turn, in the estimated $X$. For these reasons we must simultaneously estimate both $X$ and $F_{k}\left(b_{k}\right)$. Combining equations (10) and (11) yields the estimation model (for $\beta=0$ )

$$
F_{i}\left(b_{k}\right)=X_{k} \tau_{k}+F_{k}\left(b_{k}\right)
$$

This is a linear model of the form $y=\phi^{T} \theta$, where $\phi$ is a vector of known variables $\phi^{T}=\left[\begin{array}{ll}\tau_{k} & 1\end{array}\right]$, and $\theta$ is a vector of the unknown parameters to be estimated $\theta^{T}=\left[\begin{array}{ll}X_{k} & F_{k}\left(b_{k}\right)\end{array}\right]$. The recursive least-squares estimator with covariance resetting, as described in [27], is used to estimate $X_{k}$ and $F_{k}\left(b_{k}\right)$ at each sampling period. Note that $\tau_{k}$ is the time measured from the beginning of the interval. At $\tau_{k}=0$ the estimation gain (covariance) matrix is reset and the estimation of $X_{k}$ and $F_{k}$ begins.

The performance of Method I was tested in digital simulation with coefficient values that correspond to the case of cutting steel with carbide tools and the cutting variable being the speed (see Appendix B). The simulated speed variations $b_{i}$, are shown in Fig. $4(a)$. The simulated resultant force is shown at the top of Fig. $4(b)$. Figure $4(b)$ also shows the "true" wear and the estimated wear, when $C$ and $W_{0}$ are assumed known. The difference between the true and the estimated wear is less than $0.05 \mathrm{~mm}$ during the entire cutting cycle; a promising result for practical applications.

3.2 Method II. The second estimation method is used when $\beta \neq 0$ and $\gamma \simeq 0$, which is suitable for the case in which $b$ 
corresponds to the depth of cut. This is the most practically important case, since machining is always performed with different depths that depend on the part geometry (see Fig. 1). The assumption $\gamma \simeq 0$ means that the variations in the depth of cut have only a small effect on the flank wear rate, which is the case in practice. In this case, the relationship defining $\Delta F_{k}$ has the form

$$
\Delta F_{k}=X b_{k}{ }^{\beta} \tau_{k}
$$

Comparing equations (14) and (15), it is apparent that the estimation problem is more complex here than in case 1 , since the two parameters $X$ and $\beta$ must now be estimated simultaneously. For estimation purposes, the cutting force $F_{i}\left(b_{k}\right)$ is sampled at a constant rate, and a slope, $S$, defined by

$$
S=\frac{\Delta F_{k}}{\tau_{k}}=X b_{k}{ }^{\beta}
$$

is estimated for each segment $k$. In principle $S$ may be calculated from $\Delta F_{k}$ at each sampling period, and then $X$ and $\beta$ can be estimated by the recursive least-squares algorithm [27]. However, direct calculation of $S$ from equation (16) causes large errors, due to instantaneous force variations. Thus, we introduce a two-step estimation method. First, $S$ is estimated by the method introduced in Section 3.1, using the model

$$
F_{i}\left(b_{k}\right)=\hat{S}_{\tau_{k}}+F_{k}\left(b_{k}\right)
$$

instead of the model given previously in equation (14). The estimated $\hat{S}$ is subsequently input, at every sampling point, into a second estimator. The second estimator uses equation (16) to calculate $X$ and $\beta$. This second estimator is also based on the ordinary least-squares estimation algorithm, which requires that the model be linear in its parameters. However, the model in equation (16) is not linear in the unknown parameters $\beta$ and $X$. To get around this problem we take logarithms of equation (16). For $S=S$ and a large signal-to-noise ratio, equation (16) may be written as

$$
\log \hat{S}=\log X+\beta \log b_{k}
$$

Equation (18) is now linear in the unknown parameters $\log X$ and $\beta$. In the second step of the estimation algorithm, $\log X$ and $\beta$ are determined using the estimated $S$ obtained from the first step. The wear rate is proportional to $X$, as given by equation (12). The estimated wear is subsequently calculated by the accumulation process given in equation (13).

The performance of the above method was tested in a simulation study (see Appendix B). The wear, however, is independent of the cutting variable $b$ since $\gamma=0$. The variable $b$ was changed between $0.6 \mathrm{~mm}$ to $2.4 \mathrm{~mm}$ in steps of $0.6 \mathrm{~mm}$ in a pattern similar to that shown in Fig. 4(a). The corresponding cutting force is shown in Fig. $5(a)$. The force signal is filtered through a low-pass filter to assure a sufficiently large signal-to-noise ratio needed to perform the transformation from equation (16) to equation (18). The values of $\log X$ and $\beta$ are then estimated, and $X$ is subsequently calculated. The variable $C W$ is estimated by the accumulation process given in equation (13).

Method II also contains an in-process reliability test: The estimation of $C W$ does not start until the estimation of $\beta$ becomes reliable. Figure $5(b)$ shows the estimated variable $\widehat{\beta}$ which is initially very sensitive to noise. Reliability of $\hat{\beta}$ is determined by an algorithm that checks the changes in the diagonal component that corresponds to $\hat{\beta}$ in the gain matrix of the recursive least-squares estimator. In the simulated case, the accumulation starts automatically at the beginning of the 5 th step (after 1.6 minutes).

The estimated wear $\hat{W}$ is shown in Fig. 5(c). Differences between the estimated values and the true values arise due to the noise sensitivity of the estimation algorithm as a result of taking logarithms in equation (18), and due to the fact that Method II assumes a constant wear rate. However, as discussed

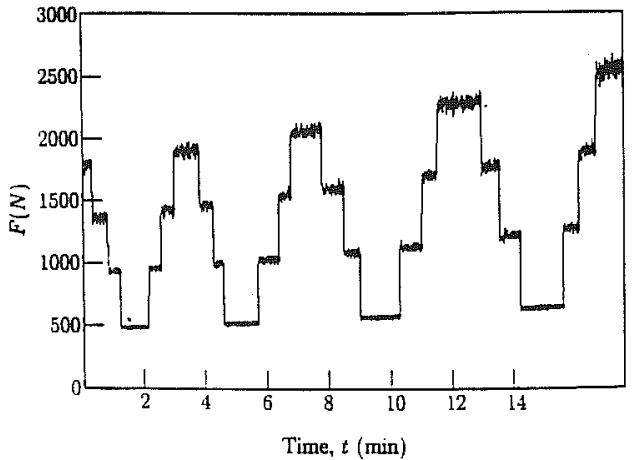

Fig. 5(a) The simulated cutting force, $F$, for method II

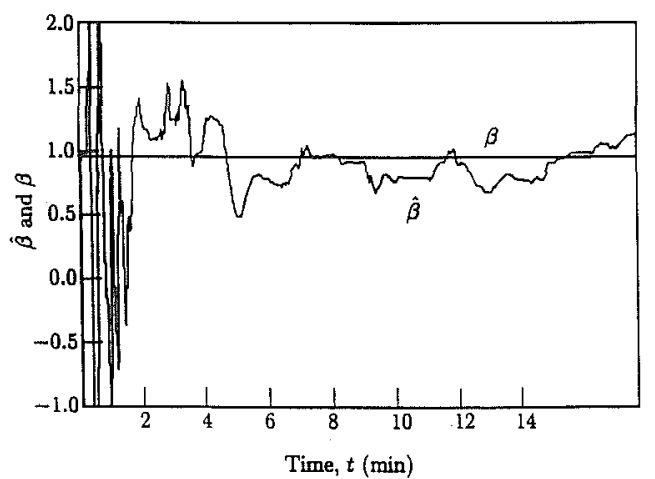

Fig. 5(b) The estimated variable $\hat{\beta}$ and the true $\beta$ in Method II

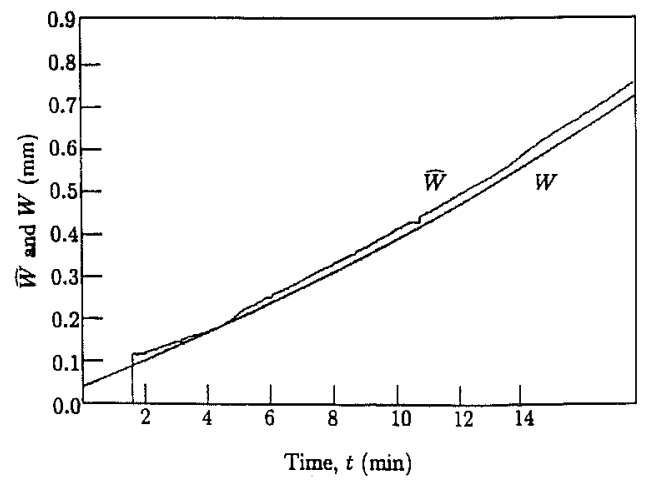

Fig. 5(c) The estimated wear, $\hat{W}$, and the true wear, $w$, for Method II

in Appendix B, the simulation includes a parabolic wear component and thus a nonconstant wear rate. The results are quite good, with wear estimation errors less than $0.04 \mathrm{~mm}$. Since we simulate realistic conditions, we expect that practical experiments will provide similar results. This expectation is confirmed by the experimental study in Section 4.

3.3 Method III. This method handles the most comprehensive case in which $\Delta F$ in equation (9) is affected by the manipulated cutting variable both directly and indirectly (through $\dot{W}$ ). In order to separate these effects, a model of the initial force $F_{0}$ must be estimated in the earlier stages of the cut (where the effect of $W$ on the cutting force is small) and used later to estimate the variable $C W$. For each of these two stages we have developed a four-step estimation algorithm.

The model of the initial measured force as derived from equations (1), (5), and (7) has the form

$$
F_{0}(b)=K_{0}^{\prime} b^{\alpha}+C b^{\beta} W^{\prime}
$$

where $K_{0}^{\prime} b^{\alpha}$, the initial force for a sharp tool, is not measurable since a small amount of wear $W^{\prime}$ is being developed during 
the first few seconds of the cut [23]. Since the term containing $W^{\prime}$ is small and $\alpha \approx \beta$ (e.g., for $b$ being the depth of cut $\alpha$ $\approx \beta \approx 1$ ), we approximate equation (19) by

$$
F_{0}(b) \approx K_{0} b^{\alpha}
$$

where $K_{0}=K_{0}^{\prime}+C W^{\prime}$. Taking logarithms of equation (20) converts it to linear form.

In order to use the least-squares estimator to estimate $K_{0}$ and $\alpha$, the algorithm requires a rich input, namely variations in $b$ during the initial period. The problem is that during this initial estimation period the tool wears, which means that the value of $K_{0}$ in equation (20) is not constant, and a conventional estimator cannot be implemented. To compensate for the wearing of the tool during the initial period, we have developed a special four-step algorithm that estimates the initial force parameters.

The estimation algorithm is based on the fact that a step change in $b$ (from $b_{k-1}$ to $b_{k}$ ) immediately affects the cutting force, but the wear at this instant remains constant (namely $K_{0}$ does not change). This means that the force before the step change is

$$
F_{k-1}^{\prime}=K_{0}(W) b_{k-1}^{\alpha_{k}}
$$

and immediately after the step is

$$
F_{k}=K_{0}(W) b_{k}^{\alpha} k
$$

where $F_{k-1}^{\prime}$ and $F_{k}$ are shown in Fig. 3. Dividing these two equations yields

$$
\alpha_{k}=\frac{\log \left(F_{k-1}^{\prime} / F_{k}\right)}{\log \left(b_{k-1} / b_{k}\right)}
$$

At each step-change in the cutting condition, a value of $\alpha_{k}$ is obtained. However, an estimation based on only one measured point (i.e., $F_{k-1}^{\prime}$ or $F_{k}$ ) is unreliable, due to measurement noise. So, we use a least-squares estimation algorithm to estimate, at each segment $k$, the initial force $\hat{F_{k}}$ and the slope $\hat{S}$ from the equation

$$
F_{i}=\widehat{F_{k}}+\widehat{S_{k}} \tau_{k}
$$

These estimated values are utilized to calculate the end-ofsegment value

$$
\hat{F_{k}^{\prime}}=\hat{F_{k}}+\hat{S_{k}} r_{k}^{\prime}
$$

where $\tau_{k}^{\prime}$ is the total time elapsed during the segment $k$. This is the first step of the algorithm. In the second step, the value of $\hat{F_{k}}$ (from equation (22)) and $\hat{F_{k-1}}$ (from equation (23) for segment $k-1$ ) are used to calculate $\alpha_{k}$ in equation (21).

Obviously $\tau_{k}^{\prime}$ is not long, since we try to keep the estimation period of the initial force model as short as possible. The estimation error in equations (22) and (23) is approximately proportional to the measured cutting force and inversely-proportional to the total observation time of a segment $\tau_{k}^{\prime}$ [28]. We have found that the inclusion of the observation time in the estimation of $\alpha$ improves substantially the reliability of the model. For this purpose, weighting factors $m_{k}$ defined by

$$
m_{k}=1 /\left(\frac{F_{k}}{\tau_{k}^{\prime}}+\frac{F_{k-1}^{\prime}}{\tau_{k-1}^{\prime}}\right)
$$

are calculated and used to estimate $\alpha$ by

$$
\hat{\alpha}=\sum_{k=2}^{n} m_{k} \alpha_{k} / \sum_{k=2}^{n} m_{k}
$$

Finally, $K_{0}$ in equation (20) is calculated by

$$
\widehat{K_{0}}=\hat{F_{1}} / b_{1}^{\alpha}
$$

with $\hat{F_{1}}$ estimated by equation (22) for the initial cutting period. (Note that $\hat{F_{1}}=\widehat{F_{0}}\left(b_{1}\right)$.)

To summarize, the estimation algorithm of the initial force $F_{0}$ consists of four steps: (1) Estimate $\widehat{F_{k}}$ and $\widehat{F_{k}}$ at each of the initial segments, (2) estimate $\alpha_{k}$ at the end of each segment,
(3) use a weighted average to obtain $\hat{\alpha}$ by equation (25), and (4) obtain $K_{0}$ by equation (26).

Once the parameters $K_{0}$ and $\alpha$ of the force model $F_{0}$ in equation (20) are identified, the model can be used for different $b$ 's during cutting. The model of $F_{0}$ with the appropriate $b$ is subtracted from the measured $F$ to compute the variable $\Delta F$ during the later stages of the cut for any cutting conditions. The $\Delta F$ obtained is subsequently used to estimate the flank wear through equation (9).

To obtain reliable results, however, the use of $\Delta F$ is not straightforward, and the estimation process requires another multi-step algorithm as explained below. If the cutting condition changes from $b_{k-1}$ to $b_{k}$ at time $t_{k}$, the force equation before the change is

$$
F_{k-1}^{\prime}=F_{0}\left(b_{k-1}\right)+C b_{k-1}^{\beta}\left(W-W_{0}\right)
$$

and after the change

$$
F_{k}=F_{0}\left(b_{k}\right)+C b_{k}^{\beta}\left(W-W_{0}\right)
$$

Namely, the change in the cutting force $\left(F_{k}-F_{k-1}^{\prime}\right)$ is attributed to two mechanisms: a change in the basic force $F_{0}$ and a change caused by the varying contact zone at the tool's flank. Note that $W$ remains constant at $t_{k}$. Using the above two relationships one can estimate $\beta_{k}$ at each step change:

$$
\beta_{k}=\frac{\log \left[\left(F_{k-1}^{\prime}-F_{0}\left(b_{k-1}\right)\right) /\left(F_{k}-F_{0}\left(b_{k}\right)\right)\right]}{\log \left(b_{k-1} / b_{k}\right)}
$$

The estimation of $\hat{C W}$ is based on another four-step algorithm, similar to the one used to estimate the initial force model, as follows:

$1 \widehat{F_{k-1}^{\prime}}$ and $\hat{F_{k}}$ are estimated by the same procedure used in the estimation of $\alpha$ in equations (22) and (23).

2 At each step, $\beta_{k}$ is calculated by equation (29), and the force change is calculated by

$$
\Delta \hat{F\left(b_{k}\right)}=\hat{F_{k}}+\hat{S_{k}} \tau_{k}-F_{0}\left(b_{k}\right)
$$

3 Weighting factors $m_{k}^{\prime}$ are assigned to each estimated $\beta_{k}$ :

$$
m_{k}^{\prime}=1 /\left(\frac{F_{k}}{F_{k}-F_{0}\left(b_{k}\right)} \frac{F_{k}}{\tau_{k}^{\prime}}+\frac{F_{k-1}^{\prime}}{F_{k-1}^{\prime}-F_{0}\left(b_{k-1}\right)} \frac{F_{k-1}^{\prime}}{\tau_{k-1}^{\prime}}\right)
$$

where the terms $F_{k} /\left(F_{k}-F_{0}\left(b_{k}\right)\right)$ and $F_{k-1}^{\prime} /\left(F_{k-1}^{\prime}-F_{0}\left(b_{k-1}\right)\right)$ are needed for normalization. Subsequently, $\beta$ is estimated by the following averaging process

$$
\hat{\beta}=\sum_{j=n}^{k} m_{j}^{\prime} \beta_{j} / \sum_{j=n}^{k} m_{j}^{\prime}
$$

4 The estimated $\hat{\beta}$ and the estimated change at the step $\Delta \hat{F\left(b_{k}\right)}$ by equation (30) are used to estimate $\hat{C W}$ from the equation:

$$
\hat{C W}=\frac{\Delta \hat{F\left(b_{k}\right)}}{b_{k}^{\beta}}
$$

As mentioned earlier, this method is sensitive to any random variations in $F_{k-1}^{\prime}$ and $F_{k}$, estimation errors, and modeling errors of $F_{0}$ (see Appendix A for a sensitivity analysis). However, as demonstrated by the simulation below, our algorithm gives good results despite this high sensitivity. It should be emphasized again that the changes in $b$ should be stepwise, and the method does not work for cases where a cutting condition is changed gradually (e.g., the depth of cut in cutting a cone on a lathe).

The performance of Method III was tested in a simulation study. The variable $b$ was changed from $0.18 \mathrm{~mm} / \mathrm{rev}$ to 0.27 $\mathrm{mm} / \mathrm{rev}$ in steps of $0.03 \mathrm{~mm} / \mathrm{rev}$ in a pattern similar to that shown in Fig. 4(a). The corresponding simulated force signal, shown in Fig. 6(a), was filtered to decrease the effect of noise on the estimation of $F_{k-1}^{\prime}$ and $F_{k}$. The two four-step estimation algorithms are then applied. The resulting estimated wear is plotted in Fig. 6(a) together with the true wear. The tool wear 


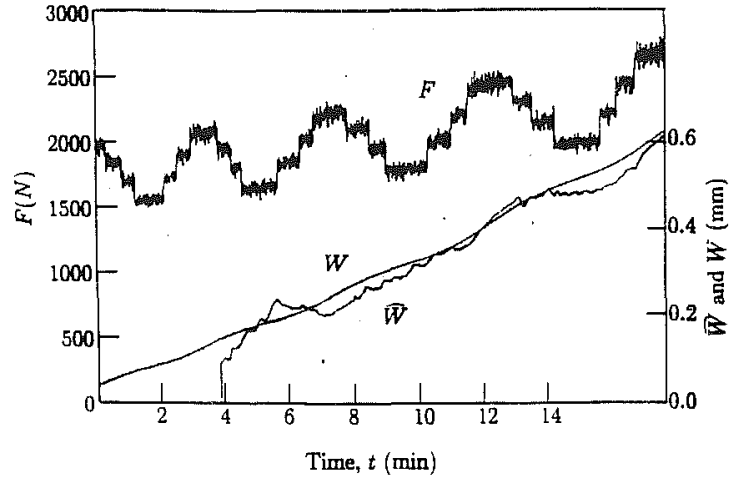

Fig. $6(a)$ The simulated cutting force, $F$, the estimated wear, $\hat{W}$, and the true wear, $W$, for Method III

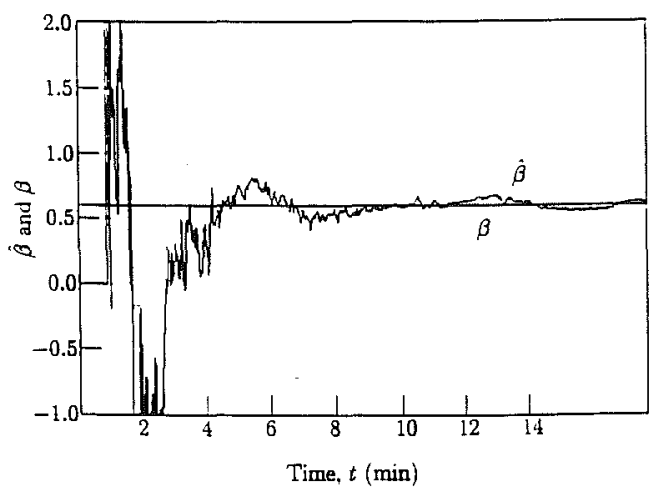

Fig 6(b) The estimated variable $\hat{\beta}$ and the true $\beta$ for Method III

estimation does not start until the estimation of $\beta$ becomes reliable. The reliability of $\beta$ is tested in real time by an algorithm that checks the magnitude of $\Sigma m_{j}^{\prime}$. The estimated $\beta$ for the simulated case is shown in Fig. $6(b)$, and tool wear estimation starts only after 3.9 minutes. This initial delay is not a problem, since in practice tools are usually changed after much longer cutting periods. As seen in Fig. 6(a), the estimated tool wear follows the true wear closely with a maximum error of 0.06 $\mathrm{mm}$. This value would be quite satisfactory for most applications.

\section{Experiments}

In order to test the performance of the estimator in practice, turning experiments were designed and performed. Only Method II was tested for changes in the depth of cut which is the most practical case, and occurs in every turning and milling process.

The methods proposed assume flank wear to be the dominant type of tool wear. Table 1 shows the cutting conditions as well as the workpiece and tool combination used. These cutting conditions were selected to generate rapid flank wear (without significant crater wear). Four tests were performed, of which three were continued until the tool failed. During these tests the depth of cut was changed in steps of $0.64 \mathrm{~mm}(0.025 \mathrm{in})$ or $1.28 \mathrm{~mm}$. Figure 7 shows the variations of the depth of cut in one of these tests and the tangential (main) component of the cutting force. The length of cut along the bar for each cutting segment was $7.62 \mathrm{~mm}(0.3 \mathrm{in})$. The tests were designed to maintain a constant cutting speed at the different diameters caused by the different depth of cuts. The actual flank wear was also measured intermittently during the tests using a Tool Makers microscope.

The experiments were carried out on a $25 \mathrm{HP}$ CNC lathe. The transducer used was a piezo-electric commercial force
Table 1 Cutting variables, tool and workpiece material

\begin{tabular}{ccccc}
\hline Test & Tool & Workpiece & Feed & $\begin{array}{c}\text { Cutting } \\
\text { speed }\end{array}$ \\
no. & TNWA & & 0.0254 & 366 \\
1 & 4340 & $\mathrm{~mm} / \mathrm{rev}$ & $\mathrm{m} / \mathrm{min}$ \\
2 & 431F & annealed & $(0.001$ & $(1200$ \\
3 & TNMA & in $/ \mathrm{rev})$ & $\mathrm{ft} / \mathrm{min})$ \\
4 & 433E & & & \\
\hline
\end{tabular}

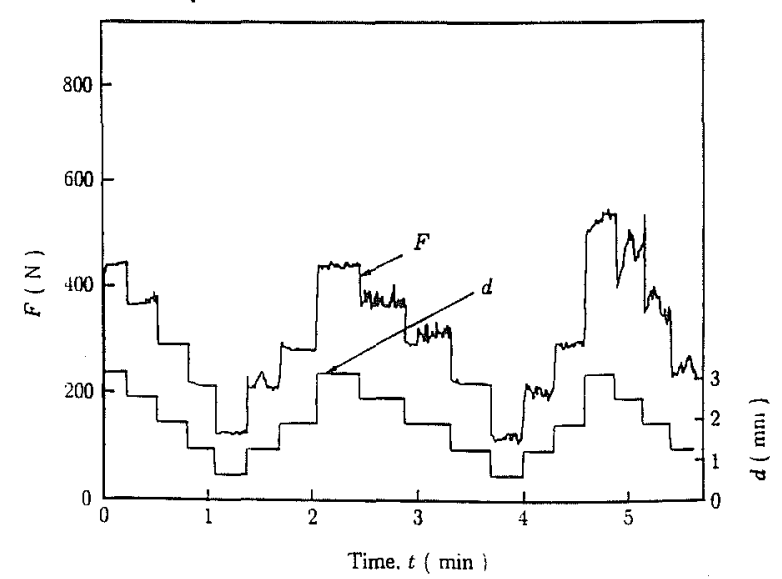

Fig. 7 The tangential cutting force component, $F$, and the depth of cut, $d$, for the 1st test

dynamometer. In order to avoid repeating the tests for signal processing purposes, the cutting force signals were recorded on an instrumentation tape recorder. A minicomputer with a 12-bit a-d convertor was used. The sampling frequency for digitization was $2 \mathrm{~Hz}$, which is sufficient to track the slow tool wear process. To avoid aliasing, low pass filters with an attenuation frequency of $1 \mathrm{~Hz}$ (half the sampling frequency) were used.

The sampled cutting force signal contained a fair amount of noise which had to be filtered. For this purpose a first order digital filter with a time constant of 2 seconds,

$$
G(z)=\frac{0.22}{z-0.78}
$$

was applied. More elaborate filter designs may give better results [29], and could be tailored to the characteristics of the turning process [30].

According to our basic assumption for tool wear estimation, the slope of the force signal versus time should be either positive or zero (for cases where tool wear stays constant). The cutting force data obtained from the above tests showed some instances where the slope was negative. Since according to our model a negative slope means an (impossible) reduction in tool wear, the periods of negative slope were taken as zero in the estimation.

The filtered cutting force data were used for tool wear estimation. The estimation results are shown in Fig. 8. To compare the estimate wear $(\hat{W})$ and the real flank wear $(W)$, direct measurements using a microscope were taken at regular intervals. Figure 9 shows $W$ versus $\hat{W}$ for seven data points.

The on-line method allows only the estimation of $(C W-$ $\widehat{C W}$ ) and not $\hat{W}$. To calculate $\hat{W}$ from $\hat{C W}$, off-line tool wear measurements are needed to determine the values of $W_{0}$ and $C$. In many cases, however, the criterion for changing tools is the start of the accelerated wear region. In these cases we are interested only in noticeable changes in the wear rate, and the off-line determination of $C$ and $W_{0}$ is not needed. 


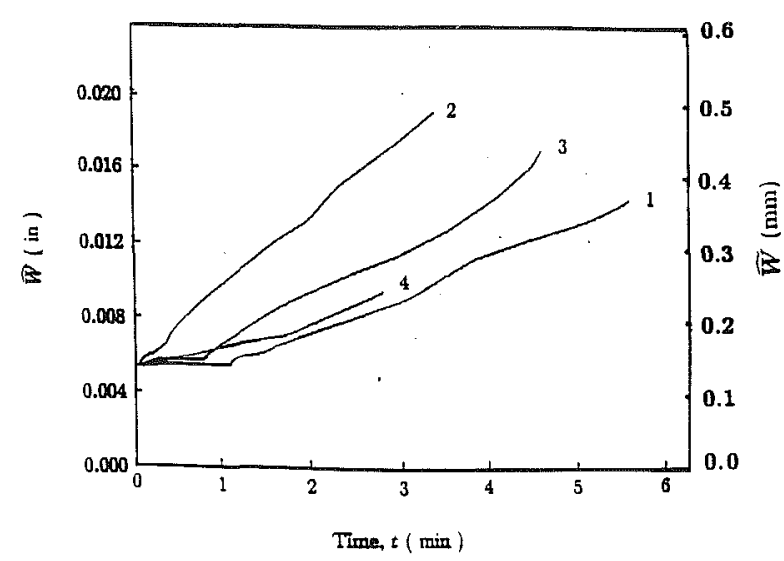

Fig. 8 Estimated wear, $\tilde{W}$ (test numbers are shown)

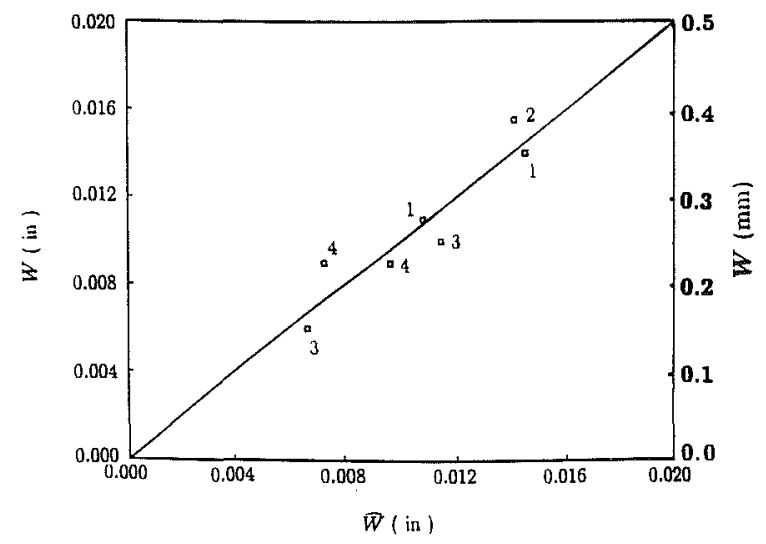

Fig. 9 Estimated wear, $\hat{W}$, versus measured wear, $W$ (data from different tests are numbered)

\section{Summary and Conclusions}

Three methods have been proposed for flank wear estimation under varying cutting conditions in turning. These methods use recursive least-squares estimation algorithms and can be applied when there are stepwise variations in one of the cutting conditions. Method I applies a standard algorithm and is included only for completeness. Methods II and III contain a real-time test of the estimation reliability and, therefore, start the wear estimation only after some initial observation period. This does not create a problem, however, since the tool wear information is needed only at the later stages of the cutting process, when the tool wear approaches some allowable limits at which on-line dimensional compensation should start, or when the tool should be replaced.

The proposed methods were all evaluated through realistic simulations (see Appendix B). The simulated force signals are very similar to those obtained in real production, since they contain noise of realistic intensity, and the wear model contains a parabolic component that simulates the accelerated wear commonly observed near the end of the tool life. This parabolic component is not included in the estimation model. The simulation results all yield very good wear estimation results, with maximum errors less than $0.06 \mathrm{~mm}$.

The wear estimates are based on the assumption of availability of the values of the intercept between the wear curve and the wear axis, $W_{0}$, and of the parameter $C$ which relates the cutting force to the tool wear. These two values may not be readily obtainable. A real-time method to obtain the values of $W_{0}$ and $C$ might be to use a few direct measurements of tool wear (e.g., with a microscope or vision system) for calibration. These measurements may be taken during loading of the workpieces. In principle, only two such measurements are needed to calibrate the estimated wear curve, but in practice more measurements are required. In some applications only the wear rate is needed, and then we do not need $C$ and $W_{0}$.

The flank wear estimates, after about the first minute of cutting are quite good in all four experiments reported, with maximum errors less than $0.05 \mathrm{~mm}$. These experimental results further reinforce the main conclusion from the simulation studies, that the proposed methods can utilize force measurement to reliably estimate flank wear in turning under varying cutting conditions. The cutting variables must vary one at a time in a stepwise constant manner and the effect of crater wear must be negligible.

\section{Acknowledgment}

The authors are pleased to acknowledge the support of the National Science Foundation (grant No. DMC 8606239) for this work.

\section{References}

1 Tlusty, J., and Andrews, G. C., "A Critical Review of Sensors for Unmanned Machining," Annals of the CIRP, Vol. 32, No. 2, 1983, pp. 563-572. 2 Wright, P. K., "Physical Model of Tool Wear for Adaptive Control in Flexible Machining Cells," presented at the ASME Winter Annual Meeting, Dec. 1984, New Orleans, LA.

3 Birla, S. K., "Sensors for Adaptive Control and Machine Diagnostics," Proceedings of the Machine Tool Task Force Conference, Vol. 4, Section 7.12, Oct. 1980.

4 Koren, Y., Computer Control of Manufacturing Systems, Chapter 8, McGraw-Hill, New York, 1983.

5 Jetly, S., "Measuring Cutting Tool Wear On-line: Some Practical Considerations," Manufacturing Engineering, July 1984, pp. 55-60.

6 Daneshmand, L. K., and Pak, H. A., "Performance Monitoring of a Computer Numerically Controlled (CNC) Lathe Using Pattern Recognition Techniques," presented at the Third International Conference on Robot Vision and Sensor Controls (ROVISEC3), Cambridge, Mass., Nov. 6-10, 1983.

7 Cook, N. H., Subramanian, K., and Basile, S. A., "Survey of the State of the Art of Tool Wear Sensing Techniques," M.I.T. report for NSF Grant No. GI-43861, Sept. 1975.

8 Jetley, S. K., "A New Radiometric Method of Measuring Drill Wear," Proceedings of the North American Manufacturing Research Conference, Houghton, Mich., May 1984, pp. 255-259.

9 De Filippi, A., and Ippolito, R., "Adaptive Control in Turning: Cutting Forces and Tool Wear Relationships for P10, P20, P30 Carbides," Annals of the CIRP, Vol. 17, 1969, pp. 377-379.

10 Bhattacharyya, A., and Ham, I., "Analysis of Tool Wear-Part 1: Theoretical Models of Flank Wear," ASME Journal of Engineering for Industry, Aug. 1969 , pp. $790-798$.

11 Koren, Y., "Differential Equation Model of a Flank Wear," CIRP Manufacturing Systems, Vol. 6, No. 1, 1977, pp. 67-73.

12 Groover, M. P., Karpovich, R. J., and Levy, E. K., "A Study of the Relationship Between Remote Thermocouple Temperatures and Tool Wear in Machining," Int. J. Prod. Res., Vol. 25, No. 2, 1977, pp. 129-141.

13 Pandit, S. M., and Kashou, S., "A Data Dependent Systems Strategy of On-Line Tool Wear Sensing," ASME Journal of Engineering for Industry, Vol. 104, Aug. 1982, pp. 217-223.

14 Kannatey-Asibu Jr., E., and Dornfeld, D. A., "A Study of Tool Wear Using Statistical Analysis of Cutting Acoustic Emission," Wear, Vol. 76, 1982, pp. 247-261.

15 Usui, E., Shirakashi, T., and Kitagawa, T., "Analytical Prediction of Cutting Tool Wear," Wear, Vol. 100, 1984, pp. 129-151.

16 Rao, S. B., "Tool Wear Monitoring Through the Dynamics of Stable Turning," ASME Journal of Engineering for Industry, Vol. 108, Aug. 1986, pp. 183-190.

17 Jiang, C. Y., Zhang, Y. Z., and Xu, H. J., "In-Process Monitoring of Tool Wear Stage by the Frequency Band-Energy Method," Annals of the CIRP, Vol. 36 , No. 1, 1987, pp. $45-48$.

18 Kronenberg, M., Machining Science \& Application, Chapter 9, Pergamon Press, 1966.

19 Armarego, E. J. A., and Brown, R. H., The Machining of Materials, Prentice-Hall, 1969.

20 Zorev, N. N., Metal Cutting Mechanics, Pergamon Press, 1966.

21 Koren, Y., "Flank Wear Model of Cutting Tools Using Control Theory," ASME Journal of Engineering for Industry, Vol. 100, No. 1, Feb. 1978, pp. $103-109$.

22 Koren, Y., "Dynamic and Static Optimization of the Cutting Processes," Proceedings of the Ist NAMR Conference, Hamilton, May 1973, Vol. 3, pp. 67-94.

$23 \mathrm{Ber}$, A., and Kaldor, S., "The First Seconds of Cutting Wear Behavior," Annals of the CIRP, Vol. 31, No. 1, 1982, pp. 13-15. 
24 Von Turkovich, B. F., "Metal Cutting: A Look Towards the Future," ASME, PED-Vol. 1, pp. 1-16, 1980.

25 Trent, E. M., "Tool Wear and Machinability," Institution of Production Engineers Journal, Mar. 1959, pp. 3-28.

26 Danai, K., and Ulsoy, A. G., "Dynamic Modeling of Cutting Forces in Turning, Drilling, and Milling from Experimental Data," Control Methods for Manufacturing Process, DSC-Vol. 9, ASME, 1988, pp. 27-34.

27 Goodwin, G. C., and Sin, K. S., Adaptive Filtering, Prediction, and Control, Prentice-Hall, Englewood Cliff, N.J., 1984.

28 Ko, T. R., Ph.D. Thesis, 1989, The University of Michigan.

29 Hamming, R. W., Digital Filters, Prentice-Hall, Englewood Cliffs, N.J., 1989.

30 Tsao, T. C., and Tomizuka, M., "Adaptive and Repetitive Digital Control Algorithms for Noncircular Machining," American Control Conference, Vol. 1,1988 , pp. $115-120$.

\section{A P P E N D I X A}

\section{Parameter Sensitivity}

This Appendix demonstrates the sensitivity of the estimated wear by Method III to error modeling in the initial force. The cutting force equation is composed of two parts: the initial force $F_{0}$ and an incremental force $\Delta F$ that is proportional to the flank wear

$$
\begin{gathered}
F=F_{0}+C b^{\beta} W \\
\Delta F=C b^{\beta} W
\end{gathered}
$$

Differentiating $W$ with respect to $F_{0}$ yields

$$
\frac{d W}{d F_{0}}=\frac{-1}{C b^{\beta}}
$$

Equation (36) can be rewritten as:

$$
\frac{d W}{W}=\frac{-F_{0}}{W C b^{\beta}} \frac{d F_{0}}{F_{0}}
$$

Substituting equation (35) into (37), we obtain

$$
\left|\frac{d W / W}{d F_{0} / F_{0}}\right|=\frac{F_{0}}{\Delta F}
$$

Equation (38) gives the sensitivity of the estimated wear $(d W / W)$ to errors in the estimation of the initial force $\left(d \hat{F_{0}}\right)$ $\hat{F_{0}}$ ). Note that for $\Delta F \sim 0$ (at $t=0$ ) the sensitivity is infinite, and even later, with a larger $\Delta F$, the sensitivity is high since $\Delta F$ is always small compared to $F_{0}$. The conclusion is that in a method that utilizes $F_{0}$ for the estimation of $W$, small errors in the measurement or estimation of $F_{0}$ cause large errors in the estimation of $W$. Despite this sensitivity problem, the estimation algorithm of Method III can provide good results.
Table 2 Parameters used in the simulations

\begin{tabular}{ccccccc}
\hline Method & $K$ & $\alpha$ & $\beta$ & $\gamma$ & $C$ & $b_{0}$ \\
\hline I & 3000 & -0.1 & 0 & 3 & 1.200 & $150 \mathrm{~m} / \mathrm{min}$ \\
II & 750 & 0.96 & 0.95 & 0 & 500 & - \\
III & 4400 & 0.64 & 0.6 & 1.5 & 2800 & $0.25 \mathrm{~mm} / \mathrm{rev}$ \\
\hline
\end{tabular}

\section{A P P E N D I X B}

\section{Details of the Simulation Studies}

The model used in the simulations is given by the following equations:

$$
\begin{aligned}
F_{0}^{*} & =K b^{\alpha}+\text { noise } \\
\Delta F^{*} & =C b^{\beta} W+\text { noise }
\end{aligned}
$$

and

$$
W=0.04+\left(0.03 t+0.0005 t^{2}\right)\left(b / b_{0}\right)^{\gamma}
$$

where the forces are in Newtons, and the flank wear $W$ is in $\mathrm{mm}$. Notice that the parabolic component in the wear model simulates the accelerated wear at large values of $t$. This parabolic component was intentionally added in the simulated wear model to account for the accelerated wear at large values of $t$. However, this accelerated wear behavior was not accounted for in the estimation method which is based on equation (2). This simulates practical situations where the wear increase is not precisely linear and the assumed model structure does not correspond to the true one. The simulated noise is white, zero-mean, and Gaussian with an amplitude of 5 percent (peak-to-peak) of the total cutting force signal $F$. This particular noise distribution has been chosen based upon observations of actual cutting forces. Equation (41) is valid for constant cutting conditions. Here, however, we assume variations in the parameter $b$, and therefore equation (41) is modified to

$$
\begin{aligned}
W=W_{0}+\sum_{i=1}^{n} \Delta t \dot{W}_{i}=0.04 & +\sum_{i=1}^{n} \Delta t(0.03 \\
& +0.001 i \Delta t)\left(\frac{b_{i}}{b_{0}}\right)^{\gamma}
\end{aligned}
$$

where $t=n \Delta t$. The estimated wear is obtained by applying a 10 -second low-pass filter to the result from the estimator.

The parameters used in equations (39) through (42) for the three methods are summarized in Table 2. These parameters correspond to the case of cutting steel with carbide tools with cutting speed in $\mathrm{m} / \mathrm{min}$, feed in $\mathrm{mm} / \mathrm{rev}$, and depth of cut in mm. 